Article

\title{
AC Transmission Emulation Control Strategies for the BTB VSC HVDC System in the Metropolitan Area of Seoul
}

\author{
Sungyoon Song ${ }^{(1)}$, Jongin Kim, Junghun Lee ${ }^{(1)}$ and Gilsoo Jang * \\ School of Electrical Engineering, Korea University, Anam-ro, Sungbuk-gu, Seoul 02841, Korea; \\ blue6947@korea.ac.kr (S.S.); uc3171@korea.ac.kr (J.K.); ejh1015@korea.ac.kr (J.L.) \\ * Correspondence: gjang@korea.ac.kr; Tel.: +82-2-3290-3246; Fax: +82-2-3290-3692
}

Received: 31 May 2017; Accepted: 31 July 2017; Published: 3 August 2017

\begin{abstract}
In the Korean power system, growing power loads have recently created the problems of voltage instability and fault current in the Seoul Capital Area (SCA). Accordingly, the back-to-back (BTB) voltage source converter (VSC) high-voltage direct-current (HVDC) system is emerging to resolve such problems with grid segmentation. However, non-convergence problems occur in this metropolitan area, due to the large change of power flow in some contingencies. Therefore, this paper proposes two kinds of AC transmission emulation control (ATEC) strategies to improve the metropolitan transient stability, and to resolve the non-convergence problem. The proposed ATEC strategies are able to mitigate possible overloading of adjacent AC transmission, and maintain power balance between metropolitan regions. The first ATEC strategy uses a monitoring system that permits the reverse power flow of $\mathrm{AC}$ transmission, and thus effectively improves the grid stability based on the power transfer equation. The second ATEC strategy emulates AC transmission with DC link capacitors in a permissible DC-link voltage range according to angle difference, and securely improves the gird stability, without requiring grid operator schedule decisions. This paper compares two kinds of ATEC schemes: it demonstrates the first ATEC strategy with specific fault scenario with PSS/E (Power Transmission System Planning Software), and evaluates the second ATEC strategy with internal controller performance with PSCAD/EMTDC (Power System Electromagnetic Transients Simulation Software).
\end{abstract}

Keywords: back-to-back HVDC; AC transmission emulation control; voltage source converter; angle difference

\section{Introduction}

In Korea, the Korea Electric Power Corporation (KEPCO) is planning to install the back-to-back (BTB) voltage source converter (VSC)-HVDC system in place of AC transmission in the metropolitan area of Seoul and its surrounding cities. The chief cause of installation is to reduce fault current magnitude through grid partitioning and to improve the metropolitan system stability. Diverse studies on VSC-HVDC systems have recently been undertaken on areas such as installation location, total capacity, bridge type, and filter capacity. However, in some contingencies, there has been a situation where the system does not converge, due to the overloading of adjacent $\mathrm{AC}$ transmission, or a voltage problem with some of the buses. Accordingly, stable operation strategies have also been actively conducted for BTB VSC-HVDC systems in metropolitan areas.

In the BTB VSC-HVDC system, two converter stations are located at the same site, and there is a short DC line between stations. Therefore, the system permits two AC grids interconnected with either the same, or a different frequency [1]. Also, the BTB VSC-HVDC system is advantageous compared to conventional $\mathrm{AC}$ transmission for the integration of renewable energy into the $\mathrm{AC}$ 
grid, or the reinforcement of regional transmission grids [2]. Furthermore, advanced technology permits the rapid and independent control of active and reactive power in all four quadrants. The control of both active and reactive power is bi-directional, and across the entire capability range [3]. In particular, converters can be situated in a weak system for voltage and frequency support, and nowadays, the system is embedded into the meshed AC grids for flexible control of power flow and fast dynamic response to several disturbances. Research conducted in the field of VSC-HVDC has included modeling [3-5] and control [6], protection [7,8], application [8,9], and reliability and evaluation $[10,11]$. Recently, power transfer research has been conducted into the modular multilevel converter (MMC) [12] and multiterminal VSC-HVDC system, which has the advantages of high reliability and flexibility in control [13,14].

There are several ongoing projects regarding the operation scheme of a BTB VSC-HVDC system. For example, the Mackinac substation controls the power order to resolve the overload situation, and supports voltage stability in the upper and lower peninsulas [15]; and in the ABB (ASEA Brown Boveri) Eagle Pass project, the BTB converter reduces the power order, and controls the DC voltage in a contingency situation. The block/unblock function and voltage dependent current order limiter (VDCOL) control are commonly applied in the industry [16]. Recently, to increase system flexibility, the use of a wide area measurement system (WAMS) method that collects voltage and current phasor information at geographically disperse locations is proposed [17]. These monitoring control schemes, which are for point-to-point HVDC systems, can effectively cope with disturbances such as power oscillations, and provide auxiliary control to the AC grid. Also, a power control strategy that changes the active power order at once in the post-disturbance period to resolve the overload problem of adjacent AC transmission is proposed in the 2015 CIGRE (International Council on Large Electric Systems) Symposium. However, the above control methods experience some drawbacks, one of which is communication delay. A monitoring control scheme for a point-to-point HVDC system naturally generates a communication time delay between distant regions, and affects short-term control in a transient situation. To resolve this problem, the grid operator should use a real-time synchronized communication device, such as a phasor measurement unit (PMU). However, since the price of a VSC-HVDC system with a real-time measurement device is high, the grid operator must consider the economics. The problem of the power control method is that the grid operator should make a schedule decision when undertaking short-range operation planning. Therefore, the operator is responsible for grid operation security under uncertain conditions.

In this paper, two kinds of AC transmission emulation control (ATEC) strategies are proposed that compensate for the problem of the existing VSC-HVDC control schemes. The ATEC strategies include an internal controller for angle difference, which improves transient stability without requiring schedule decisions in the non-convergence grid situation. For the internal controller, a mathematical model of the power equation with a monitoring system and the angle deviation versus DC voltage deviation are calculated. The proposed models are demonstrated to accurately operate in the AC grid, and effectively support the metropolitan area. This paper is structured as follows. Section 2 provides an overview of BTB VSC-HVDC system control. Section 3 discusses the installation purpose of a BTB VSC-HVDC system in the Seoul Capital Area (SCA). Section 4 proposes two operation strategies for the VSC converter. Section 5 analyzes the simulation model, and validates the proposed control strategies with PSS/E (Power Transmission System Planning Software) and PSCAD/EMTDC(Power System Electromagnetic Transients Simulation Software).

\section{BTB VSC-HVDC System Overview}

The most commonly used control approach for a VSC-HVDC system is the decoupled $\mathrm{d}$ and $\mathrm{q}$ axis control method. In this strategy, the AC currents and voltages are transformed into the rotating direct-quadrature $(\mathrm{d}-\mathrm{q})$ reference frame, and synchronized with the AC grid voltage by means of a phase-locked loop (PLL). The d-axis of the synchronously rotating reference frame is aligned to the voltage phasor, which is measured at a point of common coupling (PCC). This alignment also means 
that the q-axis component of the voltage measurement is approaching zero. This control strategy enables the decoupled control of active and reactive power and DC and AC voltage. Figure 1 shows a BTB VSC-HVDC system with a converter station (inverter) regulating DC voltage and AC voltage, with a rectifier controlling active power and AC voltage. The active power can be controlled by a power set point in the VSC-HVDC system, or indirectly regulated by DC voltage. Also, the reactive power can be regulated by a reactive power set point, or AC voltage magnitude at the PCC. The grid operator determines the control mode of a VSC-HVDC system for specific purposes. However, a BTB VSC-HVDC station in the metropolitan area commonly chooses control of the AC voltage using reactive power and fixed active power to support AC grid stability.

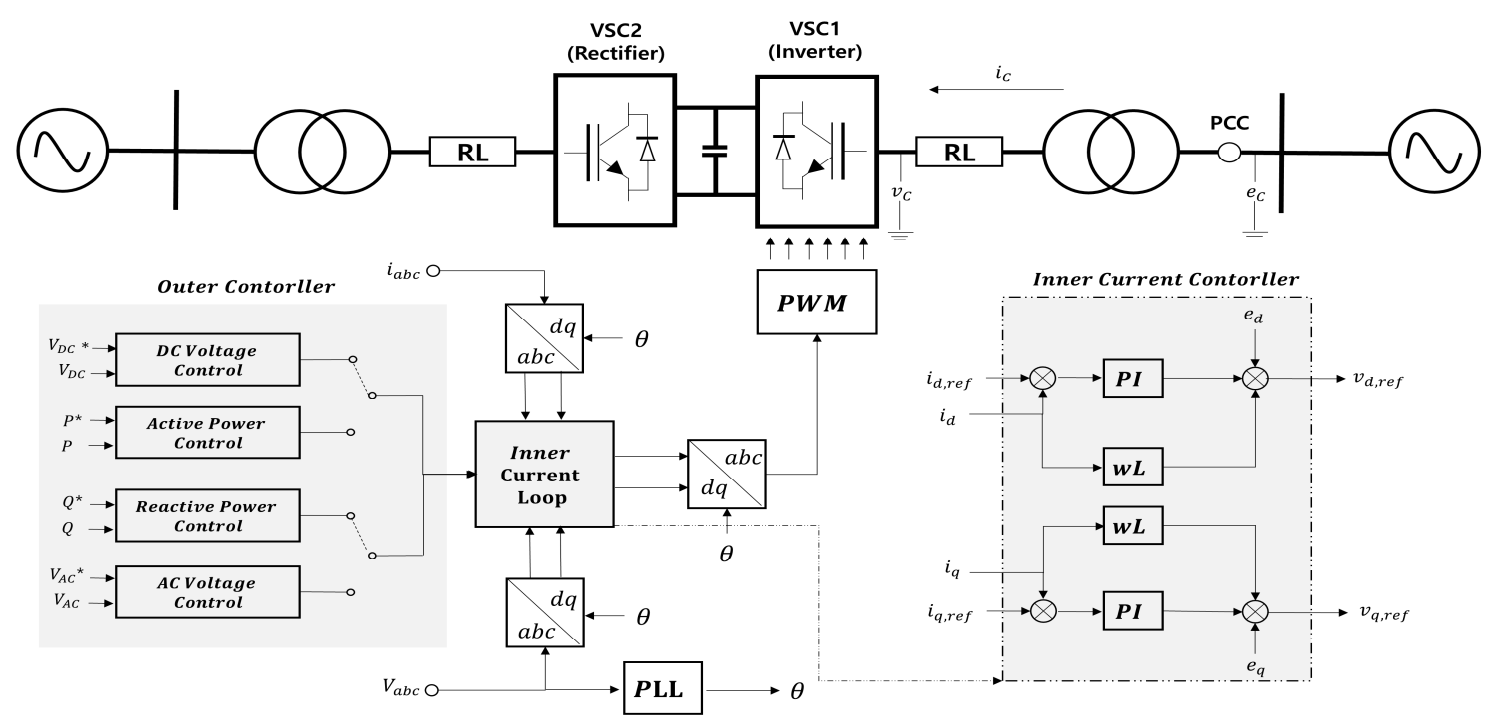

Figure 1. The voltage source converter (VSC) high-voltage direct-current (HVDC) system configuration.

Figure 1 shows the general architecture of the inner current control loop and the outer controller. The inner current control loop includes proportional integral (PI) controllers, which are much faster than outer controllers. The $e_{s}$ (voltage at PCC) and $v_{c}$ (voltage at converter) are used to produce the voltage reference for the converter:

$$
e_{s}-v_{c}=R i_{c}+L \frac{d i_{c}}{d t}
$$

where, $i_{c}$ is the current flowing from the AC grid to the converter side, and $R$ and $L$ are the resistance and inductance, respectively. Applying the d-q transform function, Equation (1) is represented by:

$$
\begin{aligned}
& e_{d}-v_{d}=R i_{d}+L \frac{d i_{d}}{d t}-\omega L i_{q}, \\
& e_{q}-v_{d}=R i_{q}+L \frac{d i_{q}}{d t}-\omega L i_{d},
\end{aligned}
$$

where, $\omega$ is the angular frequency of the AC voltage at PCC. The reference voltage $\left(v_{d, r e f}, v_{q, r e f}\right)$ generated by the inner current control loop is transformed back into the abc frame, and used for pulse with modulation (PWM) to produce the desired converter three-phase voltage.

The outer controllers permit active/reactive control and AC/DC voltage control. The active channels are activated to control the active power and DC voltage, while on the other hand, the reactive channels are responsible for reactive power and $\mathrm{AC}$ voltage control. For active and reactive power control, the power equations flowing into the AC grid are represented in the d-q reference frame as:

$$
\mathrm{P}=\frac{3}{2}\left(v_{d} i_{d}+v_{q} i_{q}\right),
$$




$$
\mathrm{Q}=\frac{3}{2}\left(v_{q} i_{d}-v_{d} i_{q}\right)
$$

However, the $\mathrm{d}$-axis of the $\mathrm{d}$-q frame is aligned with the AC system voltage phasor based on PLL, i.e., $v_{q}=0$, hence:

$$
\begin{gathered}
\mathrm{P}=\frac{3}{2}\left(v_{d} i_{d}\right) . \\
\mathrm{Q}=-\frac{3}{2}\left(v_{d} i_{q}\right) .
\end{gathered}
$$

Using the above equation, control of the active and reactive power is regulated by the decoupled $i_{d}$ and $i_{q}$ values. In particular, DC voltage variation is much faster than AC frequency variation; hence, these fast dynamic controls in the VSC converter are important in the meshed AC grid. Therefore, to maintain DC voltage at its reference value, the active power exchanged with the AC grid should be properly controlled.

\section{The BTB VSC-HVDC System Embedded in the Korea Metropolitan Area}

In common with worldwide tendencies, power loads in the Seoul Capital Area are increasing, and metropolitan transmission networks are continuously upgrading in order to meet the demand for power and replace aging local generation. However, as land space is scarce and expensive in the city, most of the power to supply load in the Seoul Capital Area should be transmitted from the east and north coastal areas. These structures cause the metropolitan area to easily encounter stability issues such as overload and voltage problems. For example, when the Seoseoul-Sinonyang line is tripped, the Seoseoul bus becomes the load concentrated end-bus, as seen in Figure 2. In the meantime, a large load in the southwestern part of the metropolitan area consumes a lot of reactive power. Accordingly, the Seoseoul bus cannot take sufficient reactive power from the metropolitan grid, which leads to the voltage instability. Therefore, the Korea Electric Power Corporation (KEPCO, Naju in South Jeolla, Korea) is in the process of reviewing technology to put the BTB VSC-HVDC system with stable operation in the Seoul Capital Area in 2030. When the BTB VSC-HVDC system is embedded in the Seoul Capital Area, several advantages will follow:

- Fault current reduction through grid segmentation; reduced circuit breaker equipment cost;

- Flexible control for the adjacent power flow margin;

- Voltage stability support in the metropolitan area;

- Power balance between regions; improvement of reliability standards.

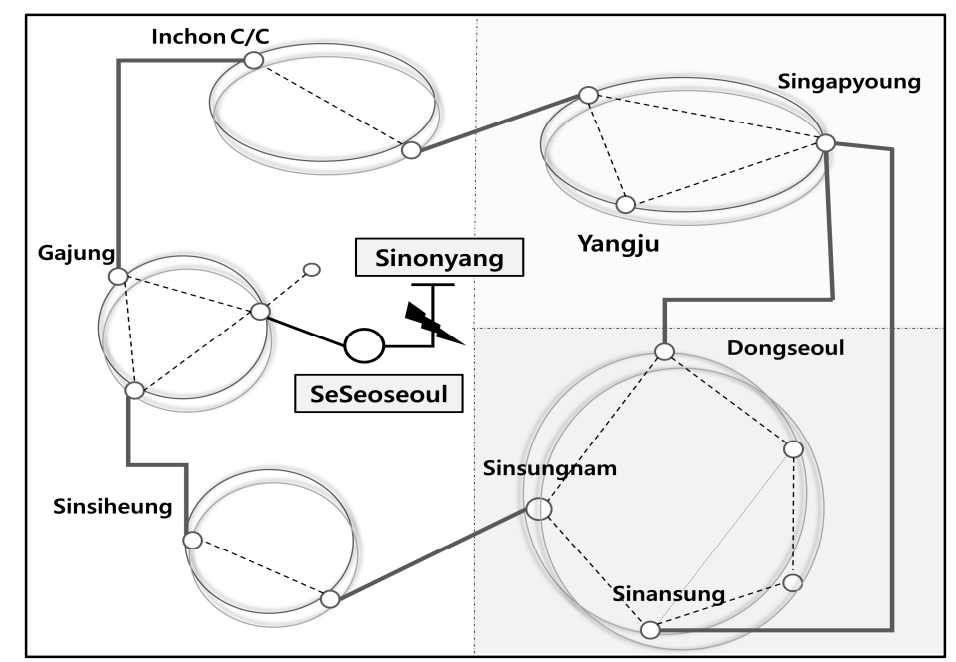

Figure 2. AC grid structure in the Korea metropolitan area. 
However, the Seoul Capital Area shows large changes in power flow direction and amount, such as reverse flow in the case of some disturbances being applied to the major AC transmission. For example, when the load capacity in Seoul is at maximum, the reliance on Incheon and the northern metropolitan area generators is increased. In this situation, to supply power to the southwestern region loads, the power flow between the Gajung-Incheon $345 \mathrm{kV}$ line is reversed in the AC system. However, if the VSC converter controls fixed power in a transient situation, the power flow of the AC grid could not be redistributed, and the grid shows non-convergence and a voltage instability situation. Also, since the impedance of fault area is very small in transient time, the nearby active power is instantaneously delivered to the fault area. In that time, if the VSC converter sustains the fixed power, the fault area that needs the immediate power cannot be delivered the power in time, which affects the subtransient frequency stability. This problem is more easily observed in Seoul area, which is a circle gird structure. Therefore, the system stability can be unstable due to clogged power flow of HVDC transmission line. Accordingly, the word "non-convergence" is newly defined in this paper as:

- Consecutive overload problem of AC transmission in power flow calculation

- Frequency instability in a sub-transient period.

To resolve the aforementioned problem, two kinds of BTB VSC-HVDC control schemes that can be synchronized with an AC system are proposed in this paper.

\section{BTB VSC-HVDC AC Transmission Emulation Control}

Due to increased load in the metropolitan area, some regional transmission lines are frequently loaded to the allowed transfer limits, and often cause reverse power flow. Accordingly, a proper BTB VSC-HVDC control strategy is needed in the metropolitan area, and the proposed ATEC strategies can help grid stabilization under various disturbances, prevent cascading outages, and counter power oscillations. The advantages of following ATEC strategies are that they:

- Mitigate the possible overloading of adjacent AC lines;

- Need not require a system operator's decision through an internal controller;

- Improve transient and post-disturbance stability;

- Follow the AC transmission tendency of reverse power flow after a specific disturbance.

\subsection{The First ATEC with Monitoring System in a BTB VSC-HVDC System}

Figure 3 shows the first strategy of the ATEC, which is to use a monitoring system to measure the nearby AC bus, which is located nearby the VSC-HVDC system. The AC bus voltage phasor is used to calculate the amount of active power from Equation (8) in the internal VSC converter, and the VSC converter makes the reference power order using the PCC voltage phasor. The AC voltage is continuously controlled by $i_{q}$ with around $1.0 \mathrm{pu}$ in a transient period, and the virtual AC transmission impedance is of fixed value; therefore, the power order is only changed by the angle difference between the sending and receiving ends. The detailed description is shown in the second ATEC strategy section, with Equation (10). The calculated power value is set to the active power order in each time step at the rectifier station (=VSC 2). This strategy permits the BTB VSC-HVDC system to follow the tendency of the AC transmission line, and it has the advantages of being intuitive and simple. Also, the system is able to follow the reverse power flow based on the power order change. Furthermore, unlike the existing control method, this strategy can be operated in a postdisturbance period, as well as a transient period. However, in the event of a severe disturbance, the power change from angle differences is so large that the system operator needs to monitor the flow of power. This strategy is the most intuitive way to imitate an AC transmission line, if the correctness of the communication signal and the appropriate limiter $\left(\mathrm{MVA}_{\text {rating }}=\mathrm{P}^{2}+\mathrm{Q}^{2}\right)$ are set. In this paper, the VSC converter controls prioritize $\mathrm{Q}$ as $1.0 \mathrm{pu}$ for metropolitan voltage stability. Therefore, active power can be increased or 
decreased, subject to the limits contained in the model. Figure 4 shows the operation range of the first ATEC strategy based on AC grid voltage (pu). The operation capability can be limited according to the $\mathrm{AC}$ grid voltage, converter voltage and $\mathrm{AC}$ grid equivalent impedance. Also, if the $\mathrm{AC}$ terminal voltage of the VSC converter is high, the reactive power output can be limited, due to the VSC converter internal voltage limit range. And, if the reactive power consumption for maintaining the voltage of a weak AC grid is large, the ability of the rectifier to follow the tendency of the AC line is limited due to $\mathrm{MVA}_{\text {rating }}=\mathrm{P}^{2}+\mathrm{Q}^{2}$. However, unlike the second ATEC strategy, this method can control both active and reactive power across the entire capability range.

In the first ATEC method, the control ability and response of the converter are very important since it generates large variation, which is sensitive to the grid operator in the system. Therefore, this paper also presents a second ATEC method. The second ATEC strategy cannot increase the system stability more than the first method, because of the small operation range. However, it does not need to involve the system operator, and it can also improve transient stability. Section 4.2 below describes the control methods in detail.

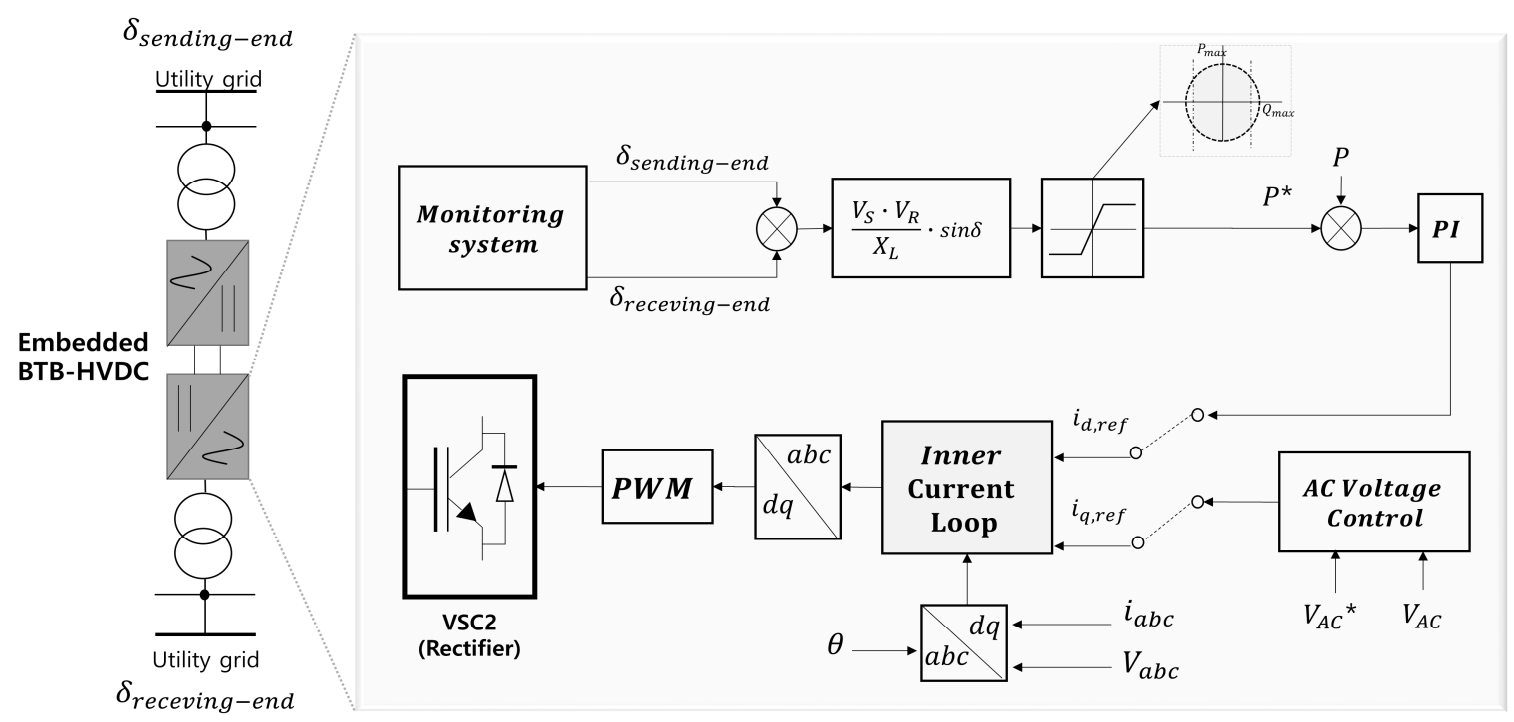

Figure 3. The first AC transmission emulation control (ATEC) strategy structure with monitoring system.
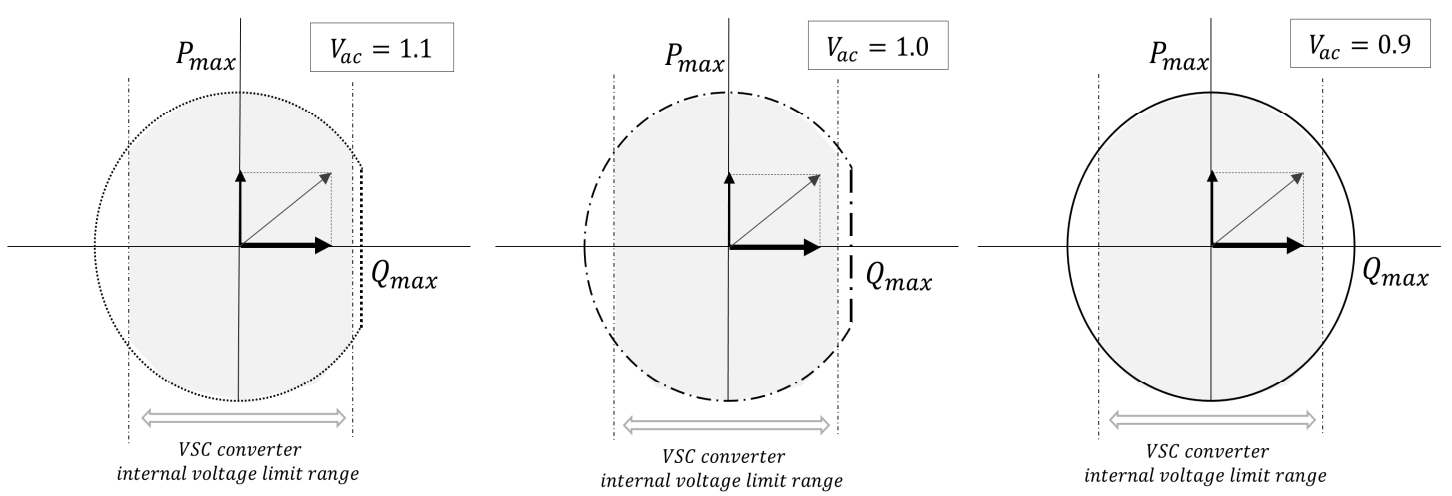

Figure 4. Operation capability of the first ATEC strategy. 


\subsection{The Second ATEC Strategy with DC-Link in a BTB VSC-HVDC System}

\subsubsection{Power Transfer Equation versus Capacitor Time}

The power transfer equation in the AC system is represented by the sending end voltage $V_{S}$, receiving end voltage $V_{R}$, and line impedance $X_{L}$ between two buses, where $\delta$ is the angle difference between the sending and receiving ends, and the power transfer function determines how much power is delivered between two buses:

$$
\mathrm{P}=\frac{V_{S} \cdot V_{R}}{X_{L}} \sin \delta,
$$

The capacitor used in the VSC-HVDC system provides energy storage to control the power flow and reduce the voltage ripple on the DC side. The size of the DC capacitor is characterized by a capacitor time constant $\tau$, and it can be expressed by the electrostatic energy $E$ stored in DC capacitors, and the VSC converter rated power capability, $S_{V S C}$, where $N$ is the total number of capacitors in the VSC-HVDC system, and $C$ is the capacitance of a DC capacitor [18]:

$$
\tau=\frac{E}{S_{V S C}}=\frac{\frac{1}{2} C N V_{D C}^{2}}{S_{V S C}}
$$

\subsubsection{Emulated AC Transmission with DC-Link in BTB VSC-HVDC}

In the $\mathrm{AC}$ transmission line, power variation over time can be expressed by $\Delta P_{1}$ in Equation (10). Actually, when a disturbance occurs in the grid, $V_{S}$ and $V_{R}$ are also changed based on time. However, due to the characteristics of the BTB VSC-HVDC system in the metropolitan area, the system commonly controls each converter side of the AC voltage within a limited converter range for improvement of metropolitan stability; therefore, the voltage variation is only slightly affected in transient time. Therefore, the voltage variation is assumed to be zero. Naturally, the VSC converter has the capability to change the $\mathrm{AC}$ voltage to emulate the $\mathrm{AC}$ transmission tendency more exactly; however, variation of the AC voltage can easily impact the stability of neighboring buses. Accordingly, BTB controls the AC voltage at around $1.0 \mathrm{pu}$ in transient time, as well as steady-state time. The $X_{L}$, which is the virtual AC line impedance in the BTB system, is of fixed value; therefore, the equation is differentiated only by the $\delta$ :

$$
\frac{V_{S} \cdot V_{R}}{X_{L}} \cos \delta \cdot \frac{d \delta}{d t}=\Delta P_{1}
$$

Equation (11) expresses the power variation of the DC capacitors. The energy in the DC capacitors is released and stored by the DC voltage variation, and those power variations can be rewritten by $\Delta P_{2}$. Since the direction of power is in direct opposition to active power flow and current flow for DC voltage control, the power variation is negative. Due to the low resistance of the DC cable, the DC voltage difference between the sending-ends and receiving-ends is negligible.

$$
\frac{C N V_{D C}}{S_{V S C}} \cdot \frac{d V_{D C}}{d t}=-\Delta P_{2}
$$

To control the $\mathrm{DC}$ voltage according to the $\mathrm{AC}$ transmission tendency, the power variations $\Delta P_{1}$ and $\Delta P_{2}$ are equated:

$$
\frac{V_{S} \cdot V_{R}}{X_{L}} \cos \delta \cdot \frac{d \delta}{d t}=-\frac{C N V_{D C}}{S_{V S C}} \cdot \frac{d V_{D C}}{d t},
$$

Integrating the equation to cancel $d \delta / d t$ and $V_{D C} / d t$, this can be written as:

$$
\begin{gathered}
\int \frac{V_{S} \cdot V_{R}}{X_{L}} \cos \delta \cdot d \delta=-\int \frac{C N V_{D C}}{S_{V S C}} \cdot d V_{D C} \\
\frac{V_{S} \cdot V_{R}}{X_{L}} \sin \delta-\frac{V_{S} \cdot V_{R}}{X_{L}} \sin \delta_{0}=-\frac{C N V_{D C}^{2}}{2 S_{V S C}}+\frac{C N V_{D C_{0}}^{2}}{2 S_{V S C}}
\end{gathered}
$$


Now, by transposing Equation (14) to active DC voltage control based on the AC transmission line tendency, the final equation to calculate the DC voltage reference is represented by Equation (15):

$$
V_{D C}=\sqrt{-\frac{2 S_{V S C}}{N \cdot C} \cdot \frac{V_{S} \cdot V_{R}}{X_{L}} \cdot\left(\sin \delta-\sin \delta_{0}\right)+V_{D C_{0}}^{2}} .
$$

Also, to find out the angle deviation based on the voltage deviation, Equation (15) can be rewritten in the form of Equation (16):

$$
\begin{gathered}
\frac{V_{S} \cdot V_{R}}{X_{L}}\left(\sin \delta-\sin \delta_{0}\right)=-\frac{C N}{2 S_{V S C}}\left(V_{D C}^{2}-V_{D C_{0}}^{2}\right), \\
\frac{V_{S} \cdot V_{R}}{X_{L}} \cdot\left(\sin \left(\Delta \delta+\delta_{0}\right)-\sin \delta_{0}\right)=-\frac{C N}{2 S_{V S C}} \cdot V_{D C_{0}}^{2} \cdot\left\{\left(\frac{\Delta V_{D C}^{2}}{V_{D C_{0}}}+1\right)^{2}-1\right\},
\end{gathered}
$$

The angle deviation can be represented by Equation (18), with the initial angle of the installation point.

$$
\Delta \delta=\sin ^{-1}\left[-\frac{C N X_{L} V_{D C}^{2}}{2 S_{V S C} V_{S} V_{R}} \cdot\left\{\left(\frac{\Delta V_{D C}^{2}}{V_{D C_{0}}}+1\right)^{2}-1\right\}+\sin \delta_{0}\right]-\delta_{0} .
$$

Figure 5 represents the angle difference deviation versus the DC voltage deviation, and each line in the graph expresses the initial angle difference between the sending and receiving ends.

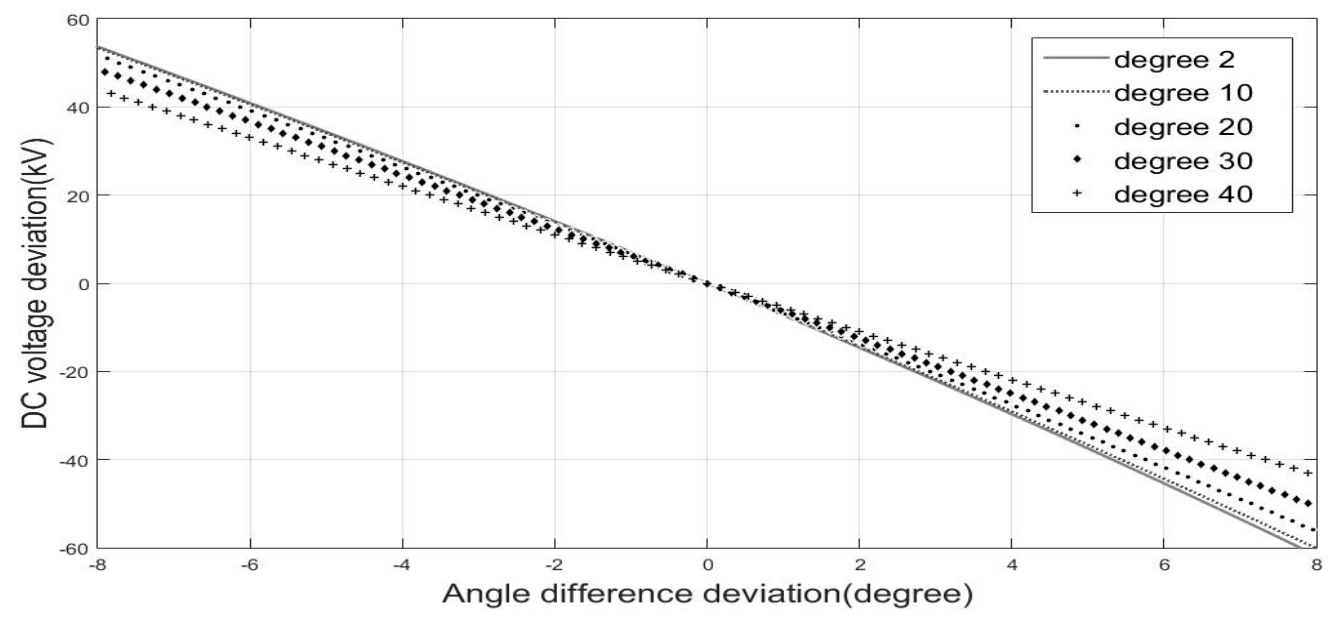

Figure 5. DC voltage deviation versus Angle difference deviation.

The variation of voltage and angle is all different, depending on the BTB VSC-HVDC installation point. For example, the initial angle difference of the dotted line is 20 degrees, which means that more power is being transmitted than 2 degrees in the AC transmission of AC system. Therefore, the larger BTB VSC-HVDC has to be installed in the location of 20 degrees to send more power. Accordingly, the Short Circuit Ratio (= Short Circuit Capacity $/ P_{d c}$ ) in the location of 20 degrees is more lower than the location of 2 degrees (Short circuit capacity is same, but $P_{d c}$ is large). In the end, the variations of voltage and angle are small, since the BTB VSC-HVDC system is located in low SCR (Short Circuit Ratio) system. 


\subsubsection{The Inner Controller of the Second ATEC Strategy with DC-Link}

Figure 6 shows the internal control logic of the second ATEC strategy. First of all, the initial angle difference $\left(=\delta_{0}\right)$ is determined by BTB VSC-HVDC installation site. Accordingly, the rate of change between the angle difference and DC voltage is determined. In the steady-state condition, the angle difference is the same, based on the power flow calculation. However, when some contingency case occurs in the AC grid, such as a bus fault or line trip, the angle difference between VSC 1 and VSC 2 is changed, based on the recalculation of the dynamic performance. Therefore, in the transient condition, the PLL estimates the angle difference, and the DC voltage reference is determined through the square root in Equation (15). Depending on the varying DC voltage, the AC transmission line acts upon the active power, which results in the enhancement of stability in the transient state.

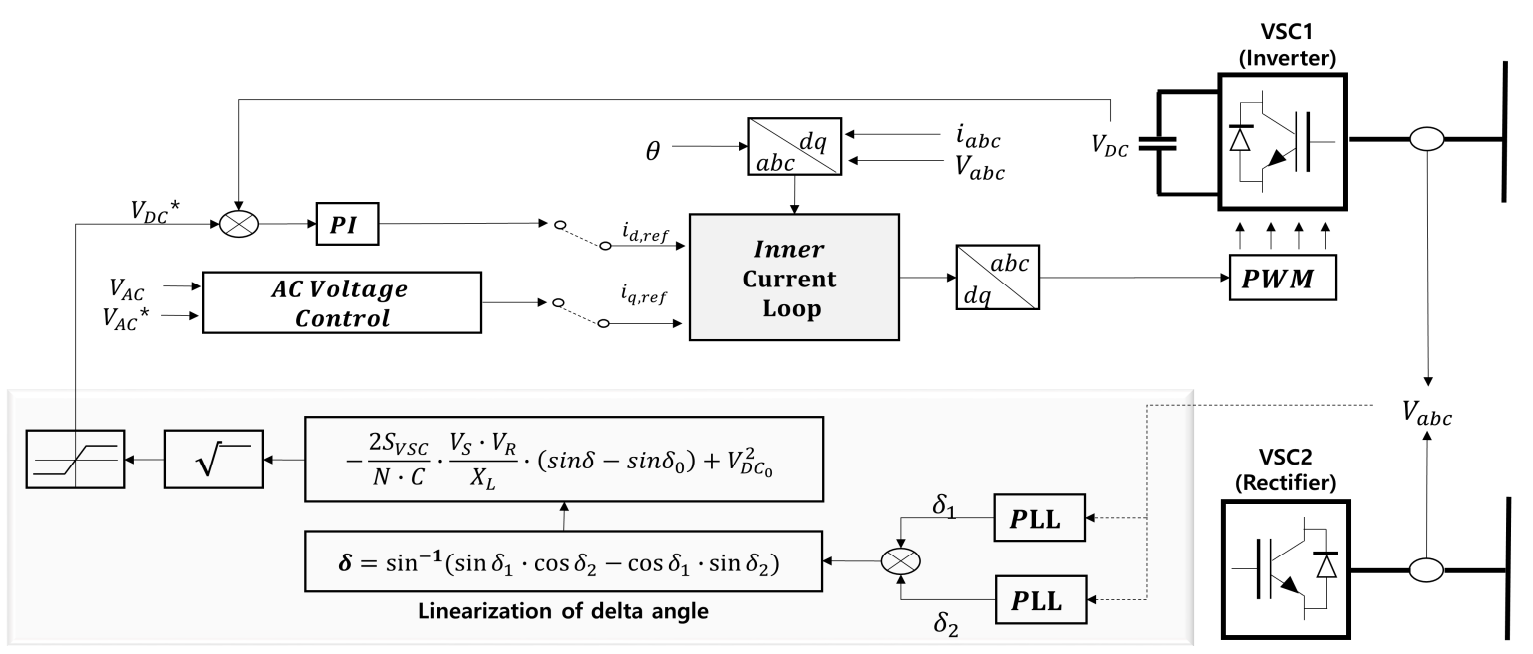

Figure 6. The second ATEC strategy structure with DC-link capacitors.

In the second ATEC strategy, there are two points to note, one of which is linearization of the delta angle. Unlike the monitoring control strategy with the first ATEC strategy, since the second ATEC strategy uses PLL, it is necessary to linearize the $\delta_{1}$ and $\delta_{2}$ in Figure 6 . Without a linearization process, a large error is created at a point where the angle difference is from 0 to 360 degree, or 360 to 0 degree. The other important point is the role of the limiter. The limiter must inject the DC voltage set point with allowable DC voltage range (commonly $\pm 15 \%$ of nominal voltage), and it can prevent large fluctuations in DC voltage coming from large angle differences after severe disturbances.

\section{Simulation Study}

\subsection{Application of the First ATEC Strategy with a Monitoring System}

To verify the first control strategy in the Korean power system, the power system dynamic analysis tool PSS/E (Siemens PTI, Berlin and Munchen, Germany) program is used with the 2030 Korea power system data. The study comprises the BTB VSC-HVDC system and the Korean power grid in Figure 7 , and Table 1 shows the detailed parameters. The BTB system embedded in the Seoul Capital Area (Seoseoul-Sinsungnam), which is connected to mesh with the AC grid, regulates the active power and AC voltage by controlling the voltage angle and amplitude. The value of the inductance and resistance is small, since the Seoul Capital Area has a large SCR (Short Circuit Ratio), and the installation site of the BTB VSC-HVDC system is determined by the degree of fault current reduction according to original purpose of the BTB installation. 


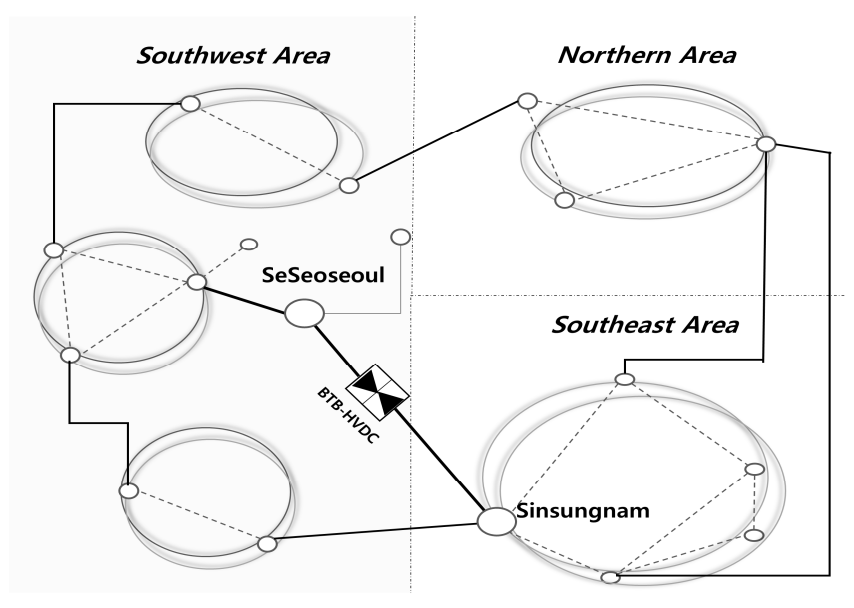

Figure 7. Installation site of the BTB VSC-HVDC system in Seoul.

Table 1. Parameters for the BTB VSC-HVDC system and AC grid.

\begin{tabular}{ccc}
\hline \multicolumn{2}{c}{ Item } & Value \\
\hline \multicolumn{2}{c}{ Rated VSC-HVDC } & $800 \mathrm{MW}$ \\
\hline \multicolumn{2}{c}{ Rated VSC-HVDC AC RMS voltage } & $345 \mathrm{kV}$ \\
\hline \multicolumn{2}{c}{ DC link Voltage } & $400 \mathrm{kV}$ \\
Installtion point of BTB & Reactor inductance & $0.00415 \mathrm{pu}$ \\
VSC-HVDC & Reactor resistance & $0.00304 \mathrm{pu}$ \\
& System frequency & $60 \mathrm{~Hz}$ \\
\hline
\end{tabular}

Using the voltage phasor of the sending and receiving ends, VSC 2 controls the active power in each time step when the angle difference is detected. To analyze the angle stability, the $\mathrm{N}-1$ contingency scenario is applied, which includes a 3-phase fault at $t=1.0 \mathrm{~s}$ in the nearby BTB VSC-HVDC installation site (=Seoseoul bus), to assume a severe contingency situation and fault clear/trip line at $t=1.1 \mathrm{~s}$. To illustrate the effectiveness of the first ATEC strategy, the simulation comprises four different control methods: the metropolitan area with only AC transmission; the metropolitan area with fixed power control of BTB (conventional BTB VSC-HVDC control); the metropolitan area with power control of BTB (proposed in the other paper [19]); and the metropolitan area with the first ATEC strategy of BTB. Figure 8 represents these individual methods. The worst case is the metropolitan area with only AC transmission, because there is no BTB converter that supports the AC voltage in the metropolitan area. The fixed power control is operated with constant power value, as shown at $395 \mathrm{MW}$ in Figure 8, whenever contingency situations occur. The existing BTB control (=the power control method) changes the power order in one time at the post-disturbance period. In contrast, in the ATEC strategy, the VSC 2 controls the active power according to the angle difference, and in this case in particular, it represents the reverse power flow tendency. The angle stability shows that among the four cases, the proposed control strategy is the most stable. Although the results may vary slightly depending on the fault scenarios, the non-convergence problem does not occur with the proposed ATEC strategy, and the grid operator need not activate a special protection system (SPS) such as a generator trip in a contingency situation. Therefore, the effectiveness of the first ATEC strategy in enhancing the stability of the metropolitan system is obvious. 

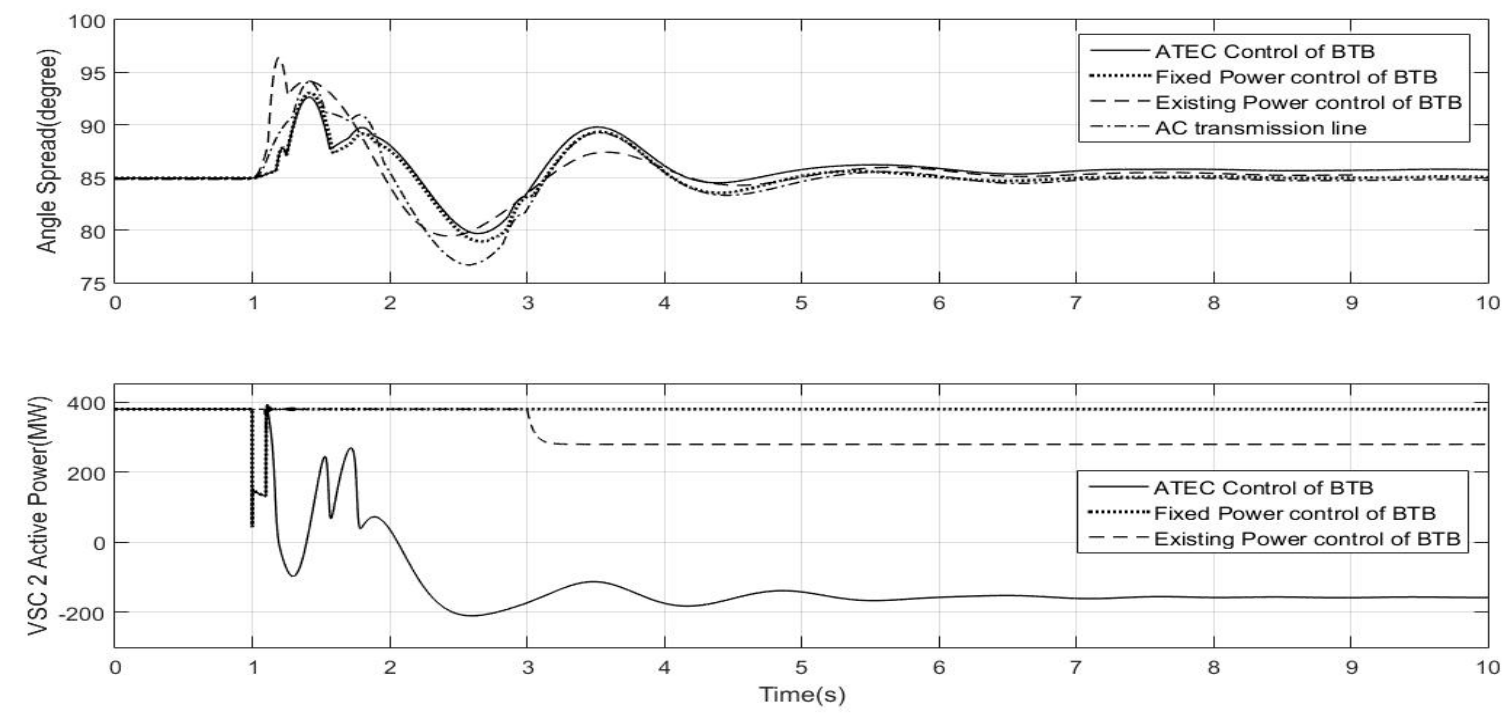

Figure 8. Angle spread and active power in the first ATEC strategy with a monitoring system.

\subsection{Application of the Second ATEC Strategy with DC-Link in BTB VSC-HVDC}

The second ATEC strategy is evaluated by using the $\pm 400 \mathrm{~V}$ BTB VSC-HVDC system, which is connected with two different AC systems in PSCAD/EMTDC (Manitoba HVDC Research Centre, Winnipeg, MB, Canada). The metropolitan area is equalized to the voltage source with high SCR. The specific parameters of the system are simply shown in schematic in the Figure 9, VSC 2 controls the active power intentionally to make the angle difference at $1.5 \mathrm{~s}$, and VSC 1 controls the DC voltage according to the sensitivity of the angle. Two scenarios are considered:

- The angle difference between the sending and receiving bus: $-\delta^{\circ}$;

- The angle difference between the sending and receiving bus: $+\delta^{\circ}$.

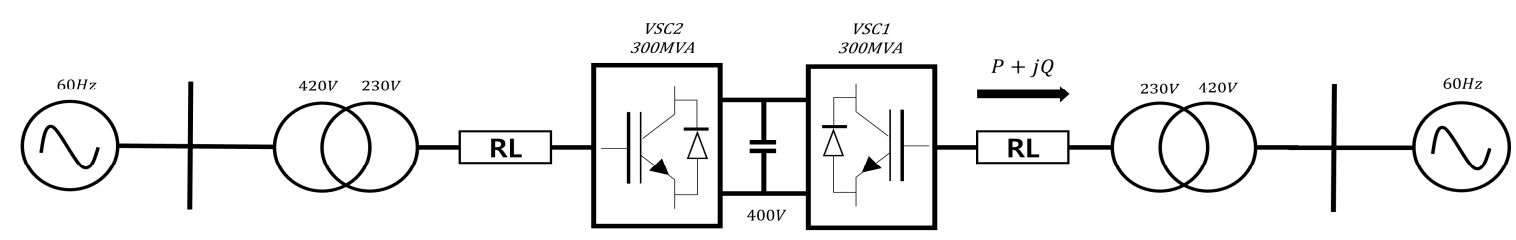

Figure 9. Single-line diagram of the test BTB VSC-HVDC system.

It must be noted that the non-convergence problem in the metropolitan area emerges, due to the transmitted power from a distant area in several contingency cases. Therefore, the equated AC voltage source in PSCAD/EMTDC cannot represent the exact situation in the Korean power system. Therefore, in this section, the simulation shows that the DC voltage is changed by the angle difference, and confirms that the active power flowing into the $\mathrm{AC}$ grid in the transient period has the same movement as the first ATEC strategy.

Figures 10 and 11 show the results of the second ATEC strategy with different angle difference using the power control of VSC 2 in detail, with negative angle variation and positive angle variation being represented, respectively. Figure $10 \mathrm{a}, \mathrm{b}$ show the change of the active power amount with an increased DC voltage, which demonstrates that the BTB VSC-HVDC system emulated the AC transmission tendency in the transient period. It is clear that the converter increases the DC voltage when the angle difference is negative, according to Equation (15). The DC capacitors store the energy across both capacitors, which increases the DC link voltage, and supports the AC grid transient stability with reduction of active power. Figure $10 \mathrm{~b}$ shows that the active power flowing into the AC grid 
is decreased, while Figure 10c shows that the active power inputs by VSC 2 remain unchanged, so that VSC 1 and VSC 2 control the active power independently, with variation of the DC link voltage. Figure 11a shows that in the second scenario $\left(+\delta^{\circ}\right)$, the DC voltage acts actively to damp the angle deviation. The DC capacitors release the energy, and this dynamic response contributes to decreased DC voltage. The damping dynamic is also attributed to the capacitance, converter-rated capacity, and line impedance, etc. Figure $11 \mathrm{~b}$ shows the increased active power flowing into the metropolitan area based on the decreased DC voltage. Therefore, the BTB VSC-HVDC system is able to emulate the AC transmission dynamic based on angle, and it can improve the power system stability of the metropolitan area like the first ATEC strategy. In both cases, the DC link voltage is maintained within the allowable voltage range $( \pm 15 \%)$. The dynamic response of the capacitors is small, since the variation of angle difference is small, as seen in Figures $10 \mathrm{~d}$ and $11 \mathrm{~d}$. Accordingly, these show that VSC 1 increases, and decreases its DC voltage slightly. If the permissible voltage range is high, the ATEC strategy could offer increased operation capability. Furthermore, the second ATEC strategy, which offers decoupling of the two-power system, has a similar stability trend to the first ATEC strategy, because the active power flowing into the AC grid helps resolve the adjacent $\mathrm{AC}$ transmission overloading problem. The first ATEC strategy, with a monitoring system, has the disadvantages that a grid operator would be involved due to large variation of the power flow, and a current saturation problem could occur due to the large angle difference. On the other hand, in the second ATEC strategy, the involvement of a grid operator is not required, and because of the DC voltage permissible range limiter, the current is not saturated. Therefore, the ATEC strategy should be determined by the grid operator considering the installation site of the BTB VSC-HVDC system, the robustness of the AC grid, the degree of instability issues, etc.
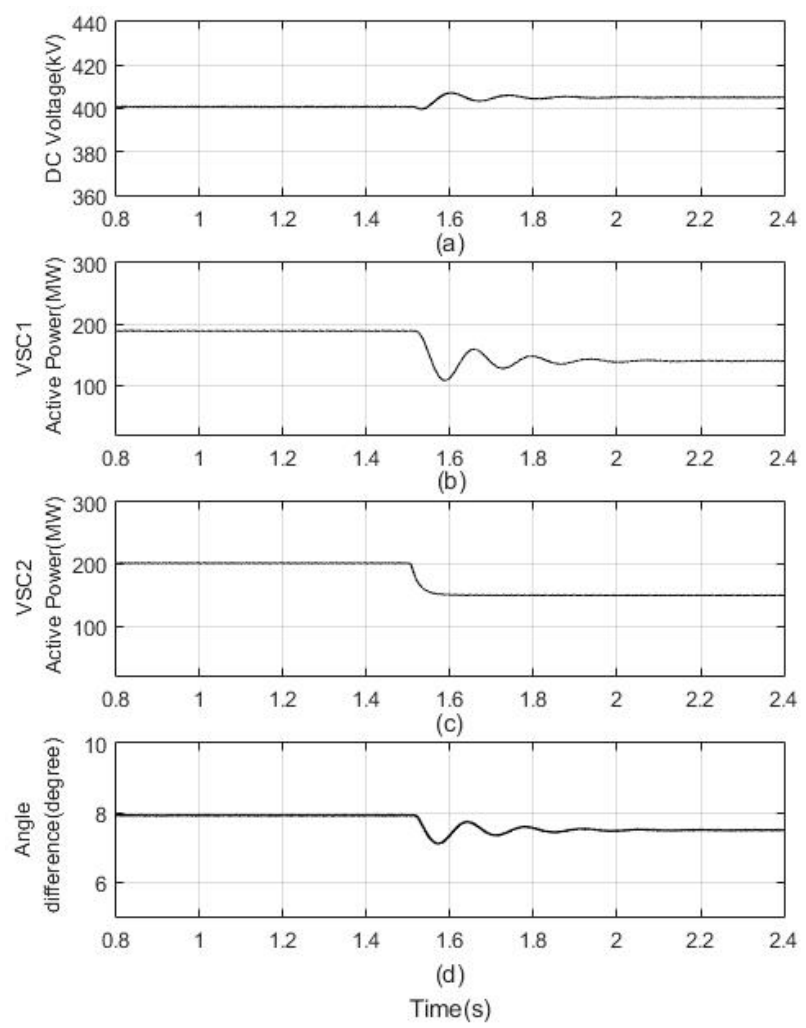

Figure 10. ATEC strategy for $-\delta^{\circ}$ scenario (a) DC voltage, (b) VSC 1 active power, (c) VSC 2 active power, and (d) angle difference. 

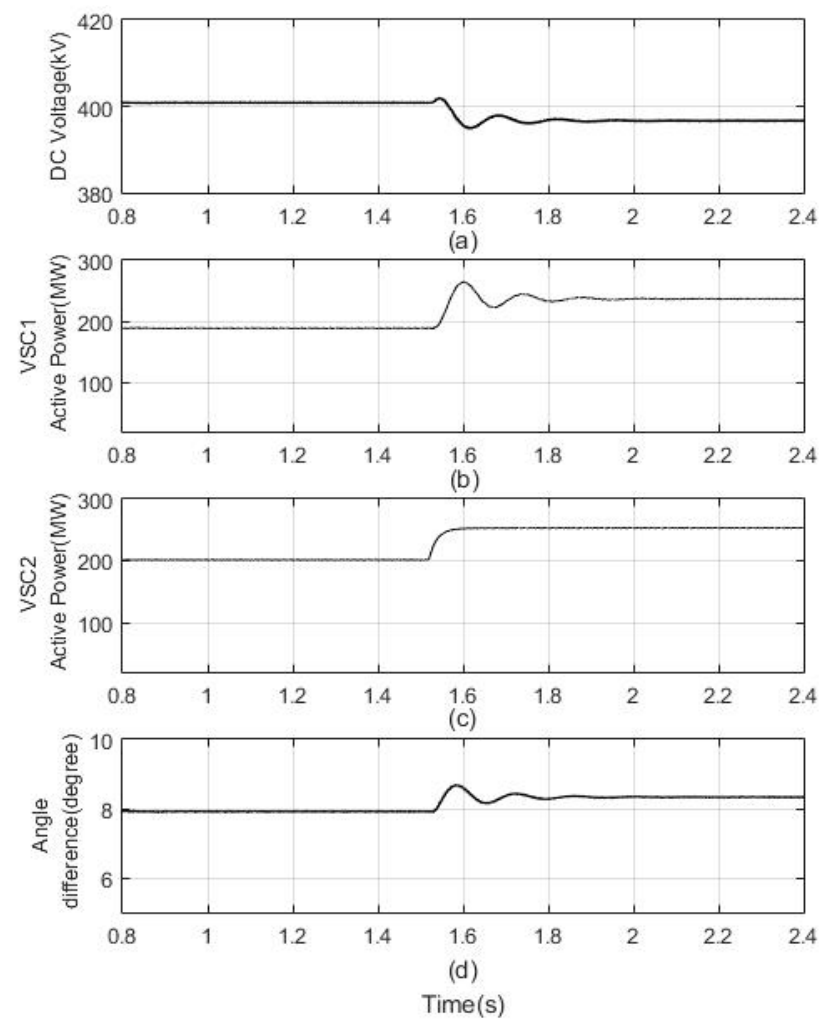

Figure 11. ATEC strategy for $+\delta^{\circ}$ scenario (a) DC voltage, (b) VSC 1 active power, (c) VSC 2 active power, and (d) angle difference.

\section{Conclusions}

This paper has proposed two kinds of AC transmission emulation control (ATEC) methods for the Seoul Capital Area in the Korean power system. The two ATEC strategies that apply a BTB VSC-HVDC system are able to improve transient stability by readjusting the active power order and by DC capacitors, respectively. The first proposed ATEC strategy changes the power order in VSC 2 based on the power transfer equation. This strategy permits a reverse power flow tendency like the AC transmission, and large operational capability within the current saturation limit. However, it has the disadvantages that due to large variability in the VSC converter station, a grid operator needs to monitor the system, and for exact control, there must be no communication delay in a transient period. The second proposed ATEC strategy regulates the DC link capacitors in VSC 1 based on angle difference within the DC voltage permissible range. The DC voltage varies with the angle difference of the sending and receiving ends; therefore, this strategy has small variability in the VSC converter, while at the same time improving the stability of the AC grid.

Through the PSS/E simulation, it is evaluated that the first proposed ATEC strategy effectively improves the transient stability in the Seoul Capital Area in 2030. The PSCAD/EMTDC simulation with the second proposed ATEC strategy briefly demonstrates that the internal controller performance is capable. In order to simulate actual non-convergence or overload conditions in PSCAD/EMTDC, it is necessary to construct an actual Korean power system, rather than simply an equivalent voltage source model. However, since the second ATEC strategy works the same as the first ATEC strategy in the transient period, it is obvious that the second ATEC strategy is more stable than general operation of the BTB VSC-HVDC system.

It is clear that BTB VSC-HVDC systems embedded in the AC grid need to react with dynamic responses in several contingency cases. Accordingly, the ATEC strategy allows the BTB VSC-HVDC system to offer decoupling control of two power systems, and more stability than fixed power control or a power control strategy that changes the active power order in the postdisturbance period at 
one time. Also, the proposed strategies enhance the control system maneuverability, and resolve the non-convergence and voltage problems in the metropolitan area.

Acknowledgments: This work was supported under the framework of international cooperation program managed by National Research Foundation of Korea (No. 2017K1A4A3013579) and the Human Resources Program in Energy Technology of the Korea Institute of Energy Technology Evaluation and Planning (KETEP) granted financial resource from the Ministry of Trade, Industry \& Energy, Republic of Korea (No. 20174030201540).

Author Contributions: The AC Transmission Emulation Control strategy in the back-to-back VSC HVDC system was proposed by Sungyoon Song and Gilsoo Jang. The experiment results were collected and analyzed by Sungyoon Song and Gilsoo Jang.

Conflicts of Interest: The authors declare they have no conflicts of interest.

\section{Nomenclature}

$\begin{array}{ll}e_{S} & \text { Voltage at PCC } \\ v_{c} & \text { Voltage at converter } \\ i_{c} & \text { Current flowing from the AC grid to the converter side } \\ \mathrm{R} & \text { Resistance } \\ \mathrm{L} & \text { Inductance } \\ \omega & \text { Angular frequency of the AC voltage at PCC } \\ v_{d, r e f} & \text { D-axis reference voltage } \\ v_{q, r e f} & \text { Q-axis reference voltage } \\ i_{d} & \text { D-axis current } \\ i_{q} & \text { Q-axis current } \\ V_{S} & \text { Sending end voltage } \\ V_{R} & \text { Receiving end voltage } \\ X_{L} & \text { Line impedance } \\ \delta & \text { Angle difference between the sending and receiving ends } \\ \delta_{0} & \text { Initial angle difference between the sending and receiving ends } \\ V_{D C} & \text { DC line voltage } \\ \mathrm{T} & \text { Capacitor time constant } \\ \mathrm{E} & \text { Electro-static energy in DC capacitors } \\ S_{V S C} & \text { VSC converter rated power capability } \\ \mathrm{N} & \text { Total number of capacitors in VSC-HVDC systems } \\ \mathrm{C} & \text { Capacitance of a DC capacitor } \\ P_{d c} & \text { HVDC power transfer }\end{array}$

\section{References}

1. Reed, G.; Pape, R.; Takeda, M. Advantages of voltage sourced converter (VSC) based design concepts for FACTS and HVDC-link applications. In Proceedings of the 2003 IEEE Power Engineering Society General Meeting, Toronto, ON, Canada, 13-17 July 2003; pp. 1816-1821.

2. Barragan, M.; Mauricio, J.M.; Marano, A.; Nieves, M.; Churio, J.; Maza-Ortega, J.M.; Romero, E.; Gómez, A. Operational benefits of multiterminal DC-links in active distribution networks. In Proceedings of the 2012 IEEE Power and Energy Society General Meeting, San Diego, CA, USA, 22-26 July 2012; pp. 1-6.

3. Fang, X.; Chow, J.H. BTB DC link modeling, control, and application in the segmentation of AC interconnections. In Proceedings of the PES'09, 2009 IEEE Power \& Energy Society General Meeting, Calgary, AB, Canada, 26-30 July 2009; pp. 1-7.

4. Tyagi, A.; Padiyar, K. Dynamic analysis and simulation of a VSC based Back-to-Back HVDC link. In Proceedings of the IICPE 2006, 2006 IEEE India International Conference on Power Electronics, Chennai, India, 19-21 December 2006; pp. 232-238.

5. Liu, Y.; Watson, N.; Zhou, K.; Yang, B. Converter system nonlinear modeling and control for transmission applications-Part I: VSC system. IEEE Trans. Power Deliv. 2013, 28, 1381-1390. [CrossRef] 
6. Parkhideh, B.; Bhattacharya, S. Resilient operation of voltage-sourced BTB HVDC systems under power system disturbances. In Proceedings of the PES'09, 2009 IEEE Power \& Energy Society General Meeting, Calgary, AB, Canada, 26-30 July 2009; pp. 1-7.

7. Farantatos, E.; Karaagac, U.; Saad, H.; Mahseredjian, J. Short-circuit current contribution of converter interfaced wind turbines and the impact on system protection. In Proceedings of the 2013 IREP Symposium Bulk Power System Dynamics and Control-IX Optimization, Security and Control of the Emerging Power Grid (IREP), Rethymno, Greece, 25-30 August 2013; pp. 1-9.

8. Larsson, T.; Petersson, A.; Edris, A.; Kidd, D.; Aboytes, F. Eagle pass back-to-back tie: A dual purpose application of voltage source converter technology. In Proceedings of the 2001 IEEE Power Engineering Society Summer Meeting, Vancouver, BC, Canada, 15-19 July 2001; pp. 1686-1691.

9. Hu, J.-B.; He, Y.-K.; Nian, H. Enhanced control of DFIG-used back-to-back PWM VSC under unbalanced grid voltage conditions. J. Zhejiang Univ. Sci. A 2007, 8, 1330-1339. [CrossRef]

10. Sato, T.; Matsushita, Y.; Temma, K.; Nakabayashi, M.; Takano, T.; Yoda, I. Concept of new BTB system and evaluation of its configuration and performance. In Proceedings of the 2002 Fifth International Conference on Power System Management and Control, London, UK, 17-19 April 2002.

11. Lee, J.H.; Yoon, M.; Jung, S.; Jang, G. System reliability enhancement in a metropolitan area using HVDC technology. J. Int. Counc. Electr. Eng. 2015, 5, 1-5. [CrossRef]

12. Chuco, B.; Watanabe, E. A comparative study of dynamic performance of HVDC system based on conventional VSC and MMC-VSC. In Proceedings of the 2010 IEEE iREP Symposium Bulk Power System Dynamics and Control (iREP)-VIII (iREP), Rio de Janeiro, Brazil, 1-6 August 2010; pp. 1-6.

13. Cole, S.; Beerten, J.; Belmans, R. Generalized dynamic VSC MTDC model for power system stability studies. IEEE Trans. Power Syst. 2010, 25, 1655-1662. [CrossRef]

14. Livermore, L.; Liang, J.; Ekanayake, J. MTDC VSC technology and its applications for wind power. In Proceedings of the 2010 IEEE 45th International Universities Power Engineering Conference (UPEC), Cardiff, UK, 31 August-3 September 2010; pp. 1-6.

15. Danielsson, J.; Hjalmarsson, P.; Karlsson, J. HVDC black start feature and its application. In Proceedings of the 2016 2nd International Colloquium on EHV and UHV, Montreal, QC, Canada, 9-11 May 2016.

16. Qian, L.; Zhong, Q.; Ma, Y.; Tao, Y.; Cao, Z. Build and validation of RTDS model for control and protection system testing of Lingbao BTB DC converter station. In Proceedings of the ICEMS 2008, IEEE International Conference on Electrical Machines and Systems, Wuhan, China, 17-20 October 2008; pp. 3867-3872.

17. Arestova, A. Application of BtB converter stations potential to improve UCTE-IPS/UPS interconnection. In Proceedings of the International Youth Conference on Energetics, Budapest, Hungary, 4-6 June 2009.

18. Zhu, J.; Booth, C.D.; Adam, G.P.; Roscoe, A.J.; Bright, C.G. Inertia emulation control strategy for VSC-HVDC transmission systems. IEEE Trans. Power Syst. 2013, 28, 1277-1287. [CrossRef]

19. Danielsson, J.S.P.; Pan, J.; Nuqui, R. Transmission grid reinforcement with embedded VSC-HVDC. In Proceedings of the US National Committee 2015, CIGRE Grid of the Future Symposium 2015, Chicago, IL, USA, 11-13 October 2015.

(C) 2017 by the authors. Licensee MDPI, Basel, Switzerland. This article is an open access article distributed under the terms and conditions of the Creative Commons Attribution (CC BY) license (http://creativecommons.org/licenses/by/4.0/). 